\title{
The APETALA2 homolog CaFFN regulates flowering time in pepper
}

\author{
Xinjie Yuan ${ }^{1,2}$, Rong Fang ${ }^{1}$, Kunhua Zhou', Yueqin Huang ${ }^{1}$, Gang Lei ${ }^{1}$, Xiaowu Wang $\mathbb{B}^{2 凶}$ and Xuejun Chen ${ }^{1 凶}$
}

\begin{abstract}
Flowering time is an important agronomic trait that contributes to fitness in plants. However, the genetic basis of flowering time has not been extensively studied in pepper. To understand the genetics underlying flowering time, we constructed an $\mathrm{F}_{2}$ population by crossing a spontaneous early flowering mutant and a late-flowering pepper line. Using bulked segregant RNA-seq, a major locus controlling flowering time in this population was mapped to the end of chromosome 2. An APETALA2 (AP2) homolog (CaFFN) cosegregated with flowering time in 297 individuals of the $\mathrm{F}_{2}$ population. A comparison between the parents revealed a naturally occurring rare SNP (SNP2T >C) that resulted in the loss of a start codon in CaFFN in the early flowering mutant. Transgenic Nicotiana benthamiana plants with high CaFFN expression exhibited a delay in flowering time and floral patterning defects. On the other hand, pepper plants with CaFFN silencing flowered early. Therefore, the CaFFN gene acts as a flowering repressor in pepper. CaFFN may function as a transcriptional activator to activate the expression of CaAGL15 and miR156e and as a transcriptional repressor to repress the expression of CaAG, CAAP1, CASEP3, CaSOC1, and miR172b based on a qRT-PCR assay. Direct activation of CaAGL 15 by CaFFN was detected using yeast one-hybrid and dual-luciferase reporter assays, consistent with the hypothesis that CaFFN regulates flowering time. Moreover, the CaFFN gene association analysis revealed a significant association with flowering time in a natural pepper population, indicating that the CaFFN gene has a broad effect on flowering time in pepper. Finally, the phylogeny, evolutionary expansion and expression patterns of CaFFN/AP2 homologs were analyzed to provide valuable insight into CaFFN. This study increases our understanding of the involvement of CaFFN in controlling flowering time in pepper, thus making CaFFN a target gene for breeding early maturing pepper.
\end{abstract}

\section{Introduction}

The timing of the transition from vegetative growth to flowering is a major factor that ensures that plants produce enough progeny for their sustainable development ${ }^{1-3}$. Thus, flowering time is an important agronomic trait that contributes to fitness in annual plants. Genetic analysis of the model plant Arabidopsis thaliana has identified numerous genes involved in flowering-time control that may participate in the photoperiod, vernalization, ambient

Correspondence: Xiaowu Wang (wangxiaowu@caas.cn) or

Xuejun Chen (19889766@163.com)

${ }^{1}$ Institute of Vegetables and Flowers, Jiangxi Academy of Agricultural Sciences, 330200 Nanchang, China

${ }^{2}$ Institute of Vegetables and Flowers, Chinese Academy of Agricultural

Sciences, 100081 Beijing, China temperature, aging, autonomous or gibberellin pathways ${ }^{4}$, each of which plays either a floral inhibiting, neutral, or promoting function in response to exogenous or endogenous signals. Downstream of these pathways are a small number of floral integrator genes, including FLOWERING LOCUS T $(F T)$ and SUPPRESSOR OF OVEREXPRESSION OF CONSTANS 1 (SOC1) $)^{5-9}$. 'Integrators' can activate floral meristem identity (FMI) genes, such as $L E A F Y(L F Y)$, APETALA1 (AP1), APETALA2 (AP2), FRUITFULL (FUL), and CAULIFLOWER (CAL), which encode proteins that promote floral development ${ }^{1}$. Among these FMI genes, the transcription factor AP2 directly promotes the expression of the floral repressor AGAMOUS-LIKE15 (AGL15) and directly represses the expression of the flowering promoting transcripts SOC1, AP1, SEPALLATA3 (SEP3),

\section{(c) The Author(s) 2021}

(c) (i) Open Access This article is licensed under a Creative Commons Attribution 4.0 International License, which permits use, sharing, adaptation, distribution and reproduction cc) in any medium or format, as long as you give appropriate credit to the original author(s) and the source, provide a link to the Creative Commons license, and indicate if changes were made. The images or other third party material in this article are included in the article's Creative Commons license, unless indicated otherwise in a credit line to the material. If material is not included in the article's Creative Commons license and your intended use is not permitted by statutory regulation or exceeds the permitted use, you will need to obtain permission directly from the copyright holder. To view a copy of this license, visit http://creativecommons.org/licenses/by/4.0/. 
AGAMOUS (AG), and $F U L^{10}$. In addition, AP2 positively regulates $m i R 156$ and negatively regulates $m i R 172$ to form a complex direct feedback loop that influences $A P 2$ expression $^{10}$.

Solanaceae includes species such as potato (Solanum tuberosum), tomato (S. lycopersicum), pepper (Capsicum annuum), eggplant (S. melongena), and tobacco (Nicotiana tabacum) that are of agricultural interest or research interest. Among these species, tomato has been more extensively studied for the control of flowering time ${ }^{11-13}$. As a member of the Solanaceae family, pepper ( $C$. annuum L.) has a sympodial shoot structure. The pepper shoot apical meristem (SAM) first produces stems and leaves alternately in the vegetative phase and then, upon the transition to the reproductive phase, terminates in an inflorescence meristem (IM) that subsequently develops into the first flower; plant growth continues from the upper most axillary meristem ${ }^{14,15}$. Flowering time in pepper can be best measured by counting the number of leaves (or nodes) on the primary stem (Nle) from the cotyledon until the first flower, which has also been described as the number of first flower nodes (FFNs) ${ }^{16-18}$.

In pepper, several genes controlling flowering time have been identified by screening an EMS-mutagenized population. Of these genes, mutants of CaJOINTLESS ( $\mathrm{CaJ}$ ), CaBLIND $(C a B L)$ and $C a S$ were late flowering ${ }^{14,15,19}$, and mutants of fasciculate ( $f a$ ) and E-62 were early flowering $^{20,21}$. These genes have a pleiotropic effect on plant growth in addition to affecting flowering time in pepper; for example, $\mathrm{CaS}$ is required for flower formation, and $C a B L$ regulates axillary meristem initiation ${ }^{15,19}$. However, most of these mutant genes are unlikely to be utilized in pepper breeding; previous studies have reported that mutants of $C a S$ were characterized by complete inhibition of flower formation ${ }^{15,19}$, and mutants of $C a B L$ had no axillary shoot development in the vegetative phase and only two sympodial units in the reproductive phase ${ }^{19}$.

Pepper germplasm resources exhibit extensive natural variation in flowering time ${ }^{22}$. Genetic analysis showed that the flowering time trait in pepper was a quantitative trait commonly controlled by a few major genes and some minor genes, as well as environmental factors such as temperature and photoperiod ${ }^{16,17,23,24}$. Quantitative trait loci (QTLs) affecting flowering time have been identified on different chromosomes using different populations ${ }^{17,18,23-26}$. For example, Tan et al. ${ }^{17}$ mapped one major and five minor QTLs affecting FFNs on chromosomes P2, P7, P10 and P11 based on an interspecific genetic map, and 12 genes were recommended as important candidate genes for the major QTL Nle2.2. Zhang et al. ${ }^{18}$ identified 23 candidate genes on chromosome 12 within an interval of $3.98 \mathrm{Mb}$ using combined specific-locus amplified fragment sequencing (SLAF-seq) and bulked segregant analysis (BSA). Zhu et al. ${ }^{26}$ detected three flowering time QTLs based on an interspecific $F_{2}$ population, in which an AP2 protein encoding gene Capana02g000700 was predicted to be the candidate gene for the major QTL. However, further studies to identify and validate the function of candidate genes controlling flowering time in pepper have been limited to date.

The early flowering pepper $\mathrm{B}_{9431}$, which can blossom and bear fruit at the first node on the main stem, is an ideal natural material for determining the genetic control of flowering time in pepper. In this study, an $\mathrm{F}_{2}$ population with 297 plants derived from an intraspecific cross between inbred lines $\mathrm{B}_{9431}$ (early flowering) and $\mathrm{A}_{145}$ (late flowering) was developed. The locus controlling flowering time in this population was mapped by the bulked segregant RNA-Seq (BSR-Seq) method. The heterologous overexpression assay in $N$. benthamiana and the virusinduced gene silencing (VIGS) assay in pepper $\mathrm{A}_{145}$ were performed to verify the function of the candidate gene CaFFN. Similarly, a real-time fluorescent quantitative PCR assay, a yeast one-hybrid assay and a dual-luciferase reporter assay were conducted to determine how the CaFFN gene influences the flowering time of pepper. Furthermore, CaFFN gene association analysis was performed on 167 pepper inbred lines to test the effect of this gene on the control of natural variations in flowering time. The phylogeny, evolutionary expansion and expression patterns of CaFFN/AP2 homologs were analyzed to provide useful insight into CaFFN.

\section{Results}

\section{Genetic analysis of the flowering time trait in pepper}

A natural precocious variant was discovered in our breeding work, from which a stable early flowering inbred line named $\mathrm{B}_{9431}$ was created after years of selfing and selective breeding (Fig. 1a). B $_{9431}$ can flower after the development of one leaf on the primary stem. To investigate the inheritance of flowering time in pepper, an $F_{2}$ population including 297 plants developed from a cross between $B_{9431}$ and $A_{145}$ was studied. Flowering time, in this study, was measured by counting the number of leaves on the primary stem between the cotyledon and the first flower (referred to as the number of FFNs) according to previous studies ${ }^{16-18}$. The average number of FFNs on parent $\mathrm{B}_{9431}$ was 2.3 (ranging from 1 to 4 ), parent $\mathrm{A}_{145}$ was 14 (ranging from 13 to 15 ), and the $F_{1}$ progeny was 8.5 (ranging from 8 to 10 ); that on the $F_{2}$ population ranged from 2 to 14 . The $F_{2}$ population showed a bimodal distribution (Fig. 1b). Based on a distribution of the $F_{2}$ population at the low point (number of FFNs of 5), the proportion of late-flowering (number of FFNs ranging from 6 to 14) to early flowering (number of FFNs ranging from 2 to 4$)$ peppers was $\sim 3: 1\left(\chi^{2}=1.08\right.$; $\left.p=0.30\right)$, thus indicating the presence of one strong major gene for this trait $^{27,28}$. 


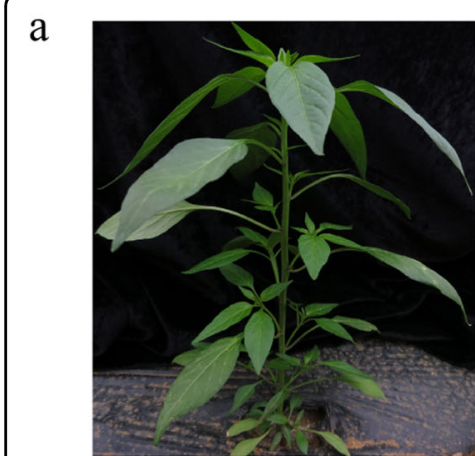

$\mathrm{A}_{145}$

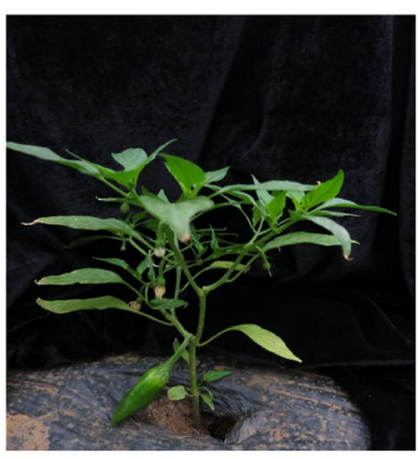

B9431

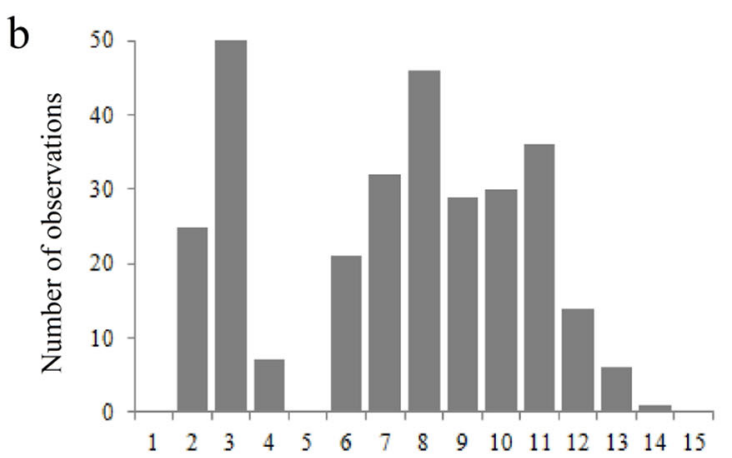

Number of first flower nodes

C

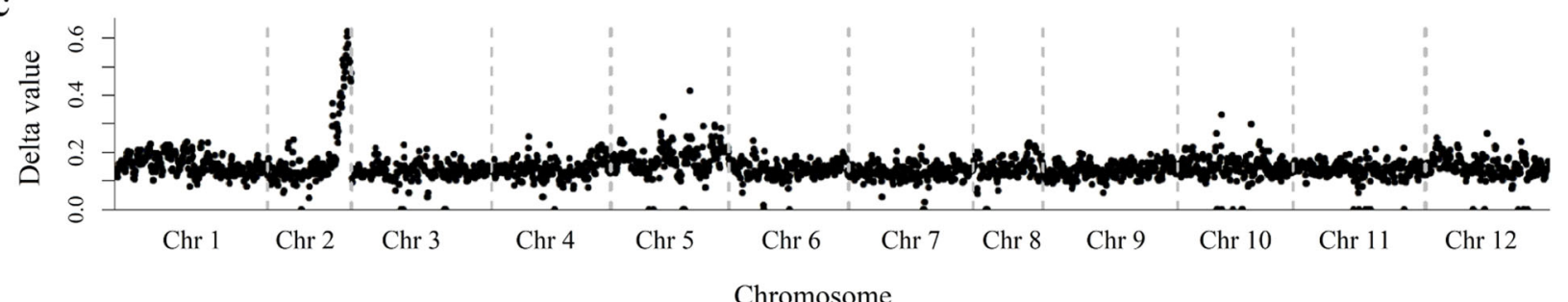

Fig. 1 Genetic analysis of the flowering time trait in pepper. a Pictures of the late-flowering parent $A_{145}$ and early flowering parent $B_{9431}$. $\mathbf{b}$ Frequency distribution of flowering time (number of first flower nodes) in the $F_{2}$ population. $\mathbf{c}$ Differences in SNP frequencies between the early and late-flowering pools from the $F_{2}$ population. The horizontal axis shows the 12 chromosomes in pepper. The vertical coordinate represents the difference in SNP frequencies between the two pools. A peak SNP value at the end of chromosome 2 suggests that one strong major locus contributes to the variation in flowering time in this population

\section{BSR-Seq identified the major locus underlying flowering time in pepper}

Thirty plants with the minimum number of FFNs from the $F_{2}$ population were chosen to construct an 'early flowering pool', and 30 plants with the maximum number of FFNs were chosen to construct a 'late-flowering pool'. The total RNA of the two pools was extracted for Illumina high-throughput sequencing, and the clean data were aligned to the C. annuum cv. CM334 genome chromosomes (release 1.55) (http://peppergenome.snu.ac.kr/). The frequencies for the single-nucleotide polymorphisms (SNPs) thus detected were calculated for each pool. The average difference in the SNP frequency (index) between the two pools was calculated across a $1.0 \mathrm{Mb}$ genomic interval using a $3.0 \mathrm{Mb}$ sliding window, and the results were plotted against all twelve chromosomes of the reference genome (Fig. 1c). The figure shows an obvious peak in the SNP delta values at the distal end of chromosome 2, which indicates one strong major locus governing flowering time in this region.

Identification of a candidate gene with a point mutation at the start codon in the early flowering parent $\mathrm{B}_{\mathbf{9 4 3 1}}$

At the end of chromosome 2, the AP2 transcription factor-encoding gene CA02g14540 caught our attention, since its likely ortholog AP2 in Arabidopsis has numerous functions, including participation in floral transition and development. The sequences of this gene in the two parents of the $F_{2}$ population were determined by sequencing the PCR products amplified with the primers listed in Supplementary Table S1. A point mutation at the start codon ATG (referred to as SNP2T $>$ C) was discovered in the early flowering parent $\mathrm{B}_{9431}$ (Fig. 2a). This SNP led to the loss of the original start codon of CaFFN and resulted in a putative frameshift mutation of CaFFN in $\mathrm{B}_{9431}$. To investigate the potential of this gene to be a candidate gene controlling flowering time in this study, a cleaved amplified polymorphic sequence (CAPS) marker CSF2 (Fig. 2b, Supplementary Table S1) was developed based on SNP2T $>$ C. The 297 individuals in the $F_{2}$ population of $\mathrm{B}_{9431} \times \mathrm{A}_{145}$ were genotyped using marker CSF2. The results showed that all early flowering (number of FFNs ranging from 2 to 4) plants had the same genotype as $\mathrm{B}_{9431}$, and all late-flowering (number of FFNs ranging from 6 to 14) plants had the same genotype as $\mathrm{A}_{145}$ or the $\mathrm{F}_{1}$ (Fig. 2b), indicating cosegregation of CSF2 with flowering time. These results demonstrated that CA02g14540 was a candidate gene (referred to as the CaFFN gene) underlying flowering time in the $\mathrm{F}_{2}$ population of $\mathrm{B}_{9431} \times \mathrm{A}_{145}$. 


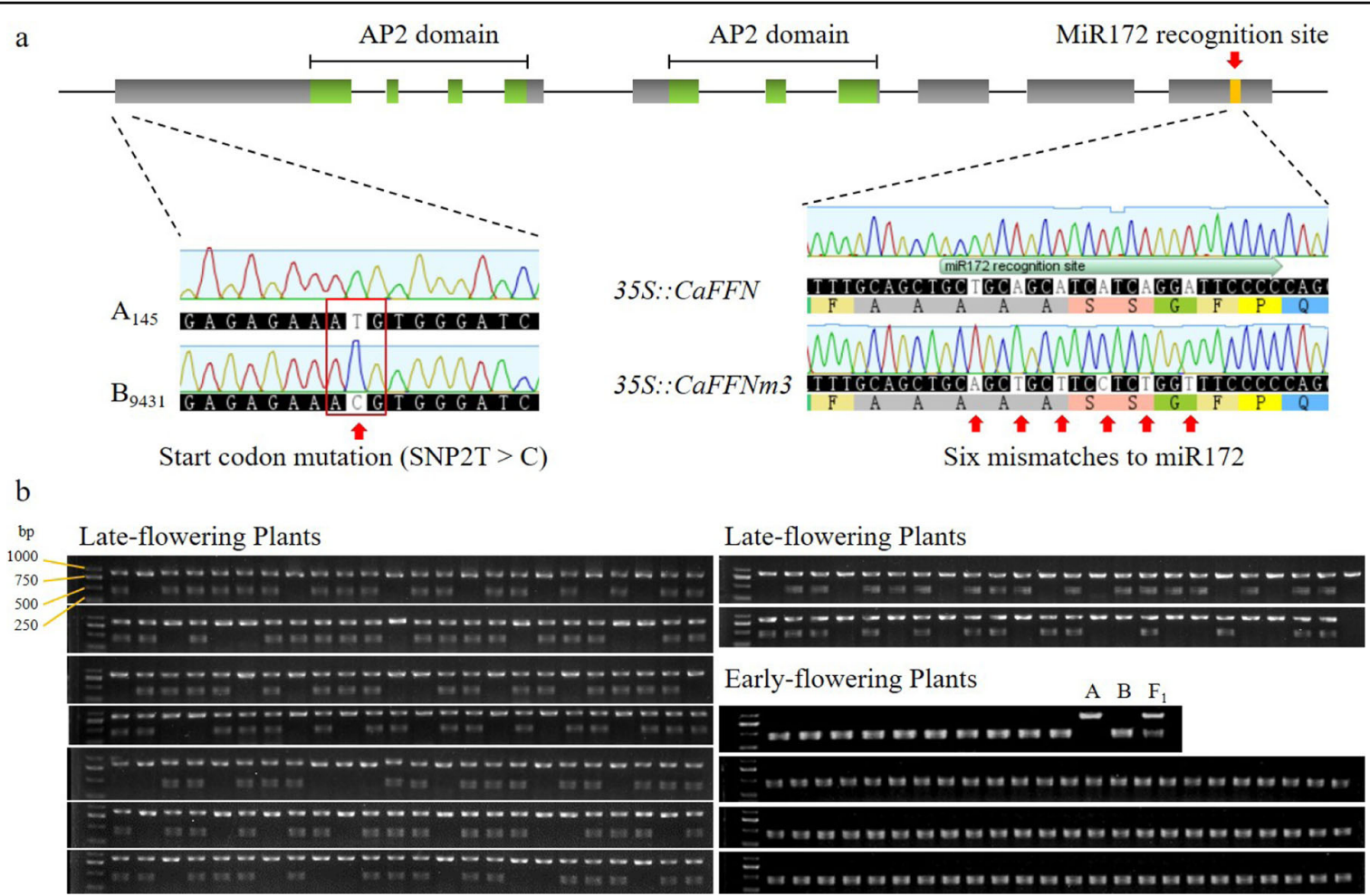

Fig. 2 The genotypes of the CaFFN gene. a Schematic representation of the CaFFN gene. The start codon of the CaFFN gene in $B_{9431}$ had a point mutation from T to C (SNP2T > C). The miR172 recognition site in 35::CaFFNm3 had six mismatches to miR172. b Marker CSF2 identified SNP2T > C through the digestion of PCR products by the restriction enzyme Tail. ' $A$ ' represents $A_{145}$, 'B' represents $B_{9431}$, ' $F_{1}$ ' represents the $F_{1}$ population of $B_{9431} \times A_{145}$, 'Late-flowering plants' represents individuals with a number of first-flower nodes (FFNs) from 6 to 14 in the $F_{2}$ population, and 'Early flowering plants' represents individuals with a number of FFNs from 2 to 4 in the $F_{2}$ population

Two $N$. benthamiana transgenic lines expressing CaFFN exhibited a notable delay in flowering time

To investigate whether CaFFN might function in controlling flowering time, transgenic $N$. benthamiana lines expressing pepper CaFFN under the control of the CaMV $35 \mathrm{~S}$ promoter were generated. However, no obvious changes in flowering time or flower patterning were observed in the 32 transgenic lines, even though the expression of the CaFFN gene was detected by qRT-PCR.

CaFFN is an AP2-like gene with a putative conserved miR172 target site. It is thus speculated that a modification of the miR172 target site would increase the expression of the CaFFN gene. For this reason, 22 transgenic lines transformed with modified CaFFN cDNA containing six mismatches (synonymous mutations) to miR172 (miR172-resistant CaFFN mutant, 35S:: CaFFNm3) were generated (Fig. 2a). Among them, two transgenic lines (lines 10 and 16) exhibited a notable delay in flowering time (Fig. 3a).

Levels of CaFFN mRNA were high in the late-flowering 35 S::CaFFNm3 lines

To further explore why these two transgenic lines behaved differently, 14 transgenic lines transformed with
35S::CaFFNm3 were used for qRT-PCR analysis. Primers FFN-RT-jF4 and CaFFN-sNR2 (Supplementary Table S1) specific to CaFFN were designed to examine the expression level of CaFFN in $N$. benthamiana. The results revealed that the expression level of the CaFFN gene was higher in these two phenotypic lines than in other plants (Fig. 3c). These two transgenic lines produced numerous leaves in an extended period of vegetative growth and flowered two and a half months later than control plants. In addition to the delayed-flowering phenotype, sterility and floral patterning defects, which were characterized by shorter filaments and poorly developed petals with a reduced size, were observed in line 10 (L10) and line 16 (L16) (Fig. 3b). L16, with the highest level of CaFFN mRNA, exhibited the most dramatic change; it produced the smallest petals and an even shorter style (Fig. 3b). These results provide evidence that the CaFFN gene has a function in regulating flowering time and floral development.

\section{Silencing of the CaFFN gene promotes early flowering in pepper}

To study the functional roles of CaFFN in flowering time in pepper, loss-of-function experiments were 


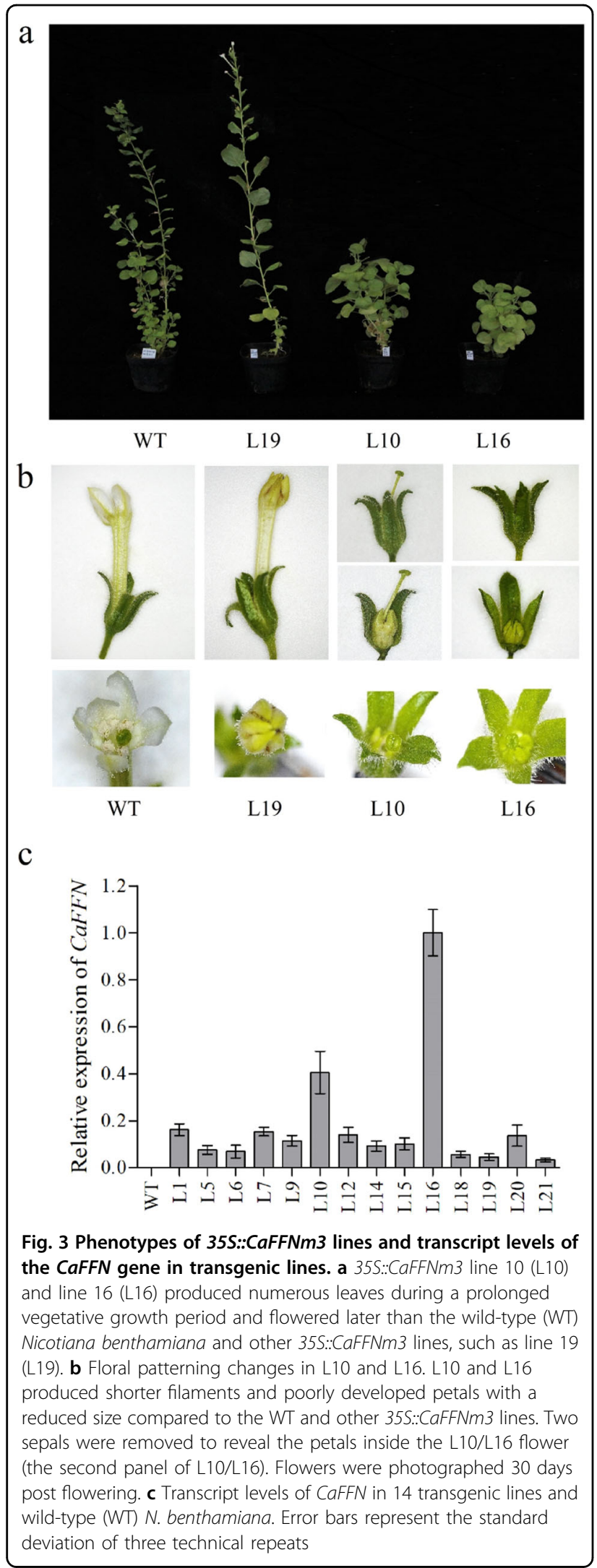

performed on parental line $\mathrm{A}_{145}$, in which CaFFN was silenced by VIGS. Agrobacterium carrying the tobacco rattle virus (TRV)-based vector TRV2::CaFFN mixed with Agrobacterium carrying the TRV1 vector were infiltrated into pepper seedlings at the four-leaf stage. Seedlings injected with TRV2::PDS- and TRV1-containing Agrobacterium were used as controls to measure the effect of gene silencing, which induced a photobleaching phenotype. Seedlings injected with TRV2- and TRV1-containing Agrobacterium were used as negative controls. The infiltrated seedlings were grown at $20 \pm 1{ }^{\circ} \mathrm{C}$ and $90 \pm 5 \%$ relative humidity to enhance silencing efficiency. Plants injected with TRV2::PDS showed photobleaching (Fig. 4a), suggesting successful gene silencing in pepper $A_{145}$. Plants injected with TRV2::CaFFN flowered earlier (the average number of FFNs was eight, with a standard error of 0.30) than control plants (the average number of FFNs was 14, with a standard error of 0.26) (Fig. 4a). Consistently, qRT-PCR results showed an obvious decline in the expression level of CaFFN in TRV2::CaFFN plants compared to the control plants (Fig. 4b). The result that silencing of the CaFFN gene leads to early flowering in TRV2::CaFFN plants further shows the function of $\mathrm{CaFFN}$ as a flowering repressor in pepper.

\section{Gene expression changes in response to CaFFN silencing}

The above results in this study indicated that CaFFN is a repressor of flowering. However, the mechanism underlying flowering time alteration caused by $C a F F N$ remains to be determined. As the closest homolog to $A P 2$, determining whether the network surrounding $A P 2$ is conserved and applicable to CaFFN is worthy of our attention. For this purpose, the likely orthologs of $A G$, AGL15, AP1, SEP3, and SOC1 (CaAG [CA02g12980], CaAGL15 [CA01g21270], CaAP1 [CA02g27000], CaSEP3 [CA11g09730], and CaSOC1 [CA01g19500]) were searched by reciprocal best-hit analysis in the $C$. annuum $\mathrm{cv}$. CM334 genome (release 1.55) (http://peppergenome.snu. ac.kr/). The expression levels of these flowering time and floral organ development genes were analyzed by qRTPCR relative to the housekeeping gene CaGAPDH [CA03g24310]. The results showed that the expression levels of CaAG, CaAP1, CaSEP3, and CaSOC1 were significantly higher in $C a F F N$-silenced plants than in the control $(P<0.01)$. In contrast, the expression level of CaAGL15 in CaFFN-silenced plants was significantly lower than that in the control $(P<0.01)$ (Fig. 4b). The expression levels of microRNAs were determined relative to CaU6 [CA07g02150]. MiR172b exhibited an increased level and miR156e represented a reduced level in CaFFNsilenced plants (Fig. 4b). The results suggested the role of CaFFN as a transcriptional activator to promote the expression of CaAGL15 and miR156e and as a 
a
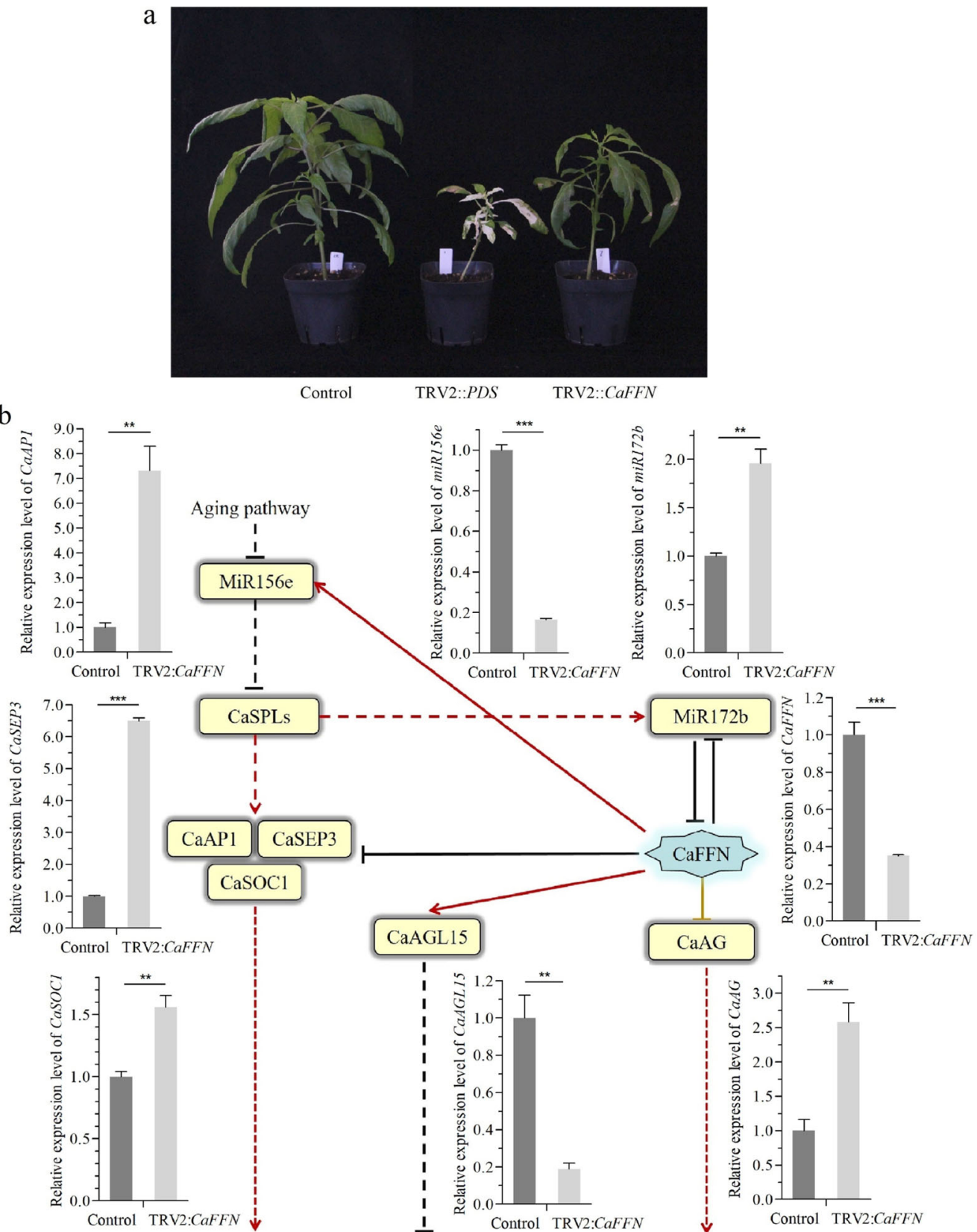

c

Flower development
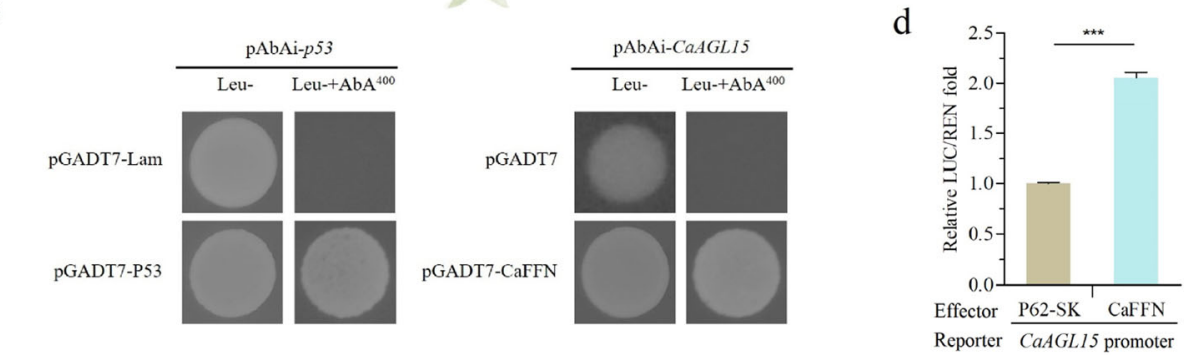

Fig. 4 (See legend on next page.) 
(see figure on previous page)

Fig. 4 Functional analysis of the CaFFN gene in pepper. a CaFFN gene silencing in $A_{145}$ via VIGS caused early flowering. TRV2::PDS plants showed photobleaching, and TRV2-CaFFN plants flowered earlier (the number of FFNs was $8 \pm 0.30$ ) than control plants (the average number of FFNs was $14 \pm 0.26)$. b Relative gene expression levels in CaFFN gene-silenced pepper plants. The expression level of the CaFFN gene was reduced via VIGS compared to the control in the shoot apical meristem at the four FFN stage. CaAG, CAAP1, CASEP3, CaSOC1, and miR172b were upregulated in CaFFNsilenced plants. CaAGL15 and miR156e were downregulated in CaFFN-silenced plants. Error bars represent the standard error of three biological replicates. Asterisks show significant differences between TRV2::CaFFN and the control (Student's $t$ test, ${ }^{* *} p<0.01,{ }^{* * *} p<0.001$ ). Hypothetical models for the transcriptional regulatory network involving CaFFN according to the transcriptional relationships in this study and reports in Arabidopsis ${ }^{10}$. Arrows represent activation, and bars represent inhibition. c CaFFN bound to the cis-elements of CaAGL15 in the yeast one-hybrid (Y1H) assay. pGADT7-p53/pAbAi-p53 and pGADT7-Lam/pAbAi-P53 were used as positive and negative controls, respectively. Superscripts represent the concentration of aureobasidin A (AbA). $\mathbf{d}$ The ability of CaFFN to bind to the promoter of CaAGL15 was tested by dual luciferase assay. The LUC/REN fold value of the empty vector P62-SK plus promoter was set to 1.0. The values are the means \pm SD (Student's $t$ test, ${ }^{* * *} p<0.001$ )

transcriptional repressor to repress the expression of CaAG, CaAP1, CaSEP3, CaSOC1, and miR172b.

\section{The CaFFN transcription factor directly activates the floral repressor gene CaAGL15}

The finding that the expression level of the floral repressor gene CaAGL15 was reduced in CaFFN genesilenced pepper plants prompted us to examine whether CaAGL15 is directly regulated by CaFFN. We checked the ability of CaFFN to bind to the cis-elements of CaAGL15 using a yeast one-hybrid assay. The full-length cDNA of CaFFN was cloned in frame with the GAL4 activation domain (AD) in the pGADT7 vector. The cis-elements of CaAGL15 were inserted into the pAbAi vector. The minimal inhibitory concentration of aureobasidin A (AbA) was $400 \mathrm{ng} / \mathrm{ml}$ for bait strains of CaAGL15. The results showed that CaFFN directly interacted with CaAGL15, since yeast transformed with pGADT7-CaFFN and the bait vector grew in $\mathrm{SD} /$-Leu selection medium supplemented with the minimal inhibitory concentration of AbA, while yeast transformed with pGADT7-lam and pAbAi-P53, as a negative control, did not (Fig. 4c). In addition, a dual-luciferase assay was performed in $N$. benthamiana leaves. Recombinant pGREEN0800CaAGL15 containing the promoter region of CaAGL15 (reporter) and the fused protein with CaFFN (effector) were coinfiltrated into leaves. Cotransformation of CaAGL15 and the CaFFN protein resulted in a twofold LUC/REN ratio compared with the control (Fig. 4d). The results indicated that CaFFN can bind to the CaAGL15 promoter in vivo and transactivate the expression of the reporter gene.

\section{CaFFN was significantly associated with flowering time in the pepper population}

In this study, the CaFFN gene was shown to be a flowering repressor in pepper. To determine the distribution of the SNP2T > C mutation of the CaFFN gene in the pepper population, the CaFFN sequences of 383 pepper accessions were surveyed in PepperPan, a pepper pangenome browser ${ }^{29}$. The results showed that SNP2T >

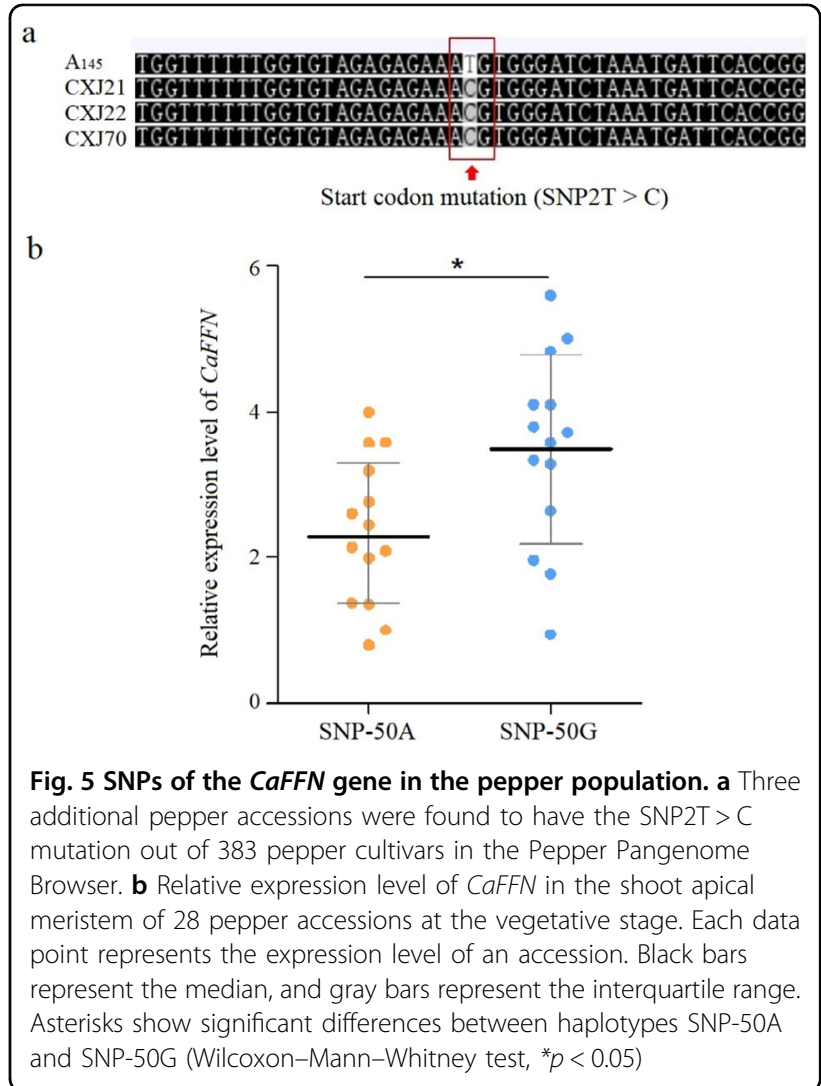

$\mathrm{C}$ was a rare SNP that occurred in only three additional accessions, CXJ21 (B33), CXJ22 (B35), and CXJ70 (A100) (Fig. 5a), and these three accessions were early flowering materials, with FFN numbers of 3, 3, and 5, respectively, suggesting that SNP2T $>C$ could affect flowering time in multiple genetic backgrounds. The rare frequency of SNP2T $>C$ also implies that the mutation occurred very recently and has not been widely used in breeding programs.

Due to the low number of accessions with nucleotide $\mathrm{C}$ at position 2, SNP2T $>C$ could not be validated by association analysis. To further investigate whether $C a F F N$ is an important regulator of flowering time in the pepper 
population, the numbers of FFNs in 164 pepper inbred lines were investigated. The numbers of FFNs exhibited wide phenotypic variations, ranging from 2 to 23, with an average of 10.5. Combined with the genotypic SNP/Indel data from the CaFFN sequenced region in 164 pepper accessions, an association analysis was performed to quantify significant associations. A SNP $50 \mathrm{bp}$ upstream of the start codon (SNP-50G $>$ A) was significantly associated with FFNs $(P=3.25 \mathrm{E}-07<0.01)$, explaining $14.5 \%$ of the phenotypic variation. The results demonstrated that CaFFN was significantly associated with flowering time in the pepper population.

A sequence comparison between the CaFFN alleles of 53 pepper accessions did not reveal clear causative polymorphisms in the coding region. It is speculated that the $5^{\prime}$-UTR may influence the number of FFNs by affecting the expression of the CaFFN gene. To test this hypothesis, 28 randomly selected accessions were divided into two haplotypes according to SNP-50G > A, and the expression level of the CaFFN gene was determined by qRT-PCR (Fig. 5b). Statistical analysis showed that SNP-50G >A significantly correlated with the expression level of the CaFFN gene $(P<0.05)$, suggesting that the expression level of CaFFN might be influenced by its $5^{\prime}$-UTR.

\section{Characterization of the CaFFN/AP2 homologs in pepper}

CaFFN has two AP2 domains and is a member of the $A P 2$ family gene. AP2 family genes with two or more AP2 domains play essential roles in plant growth and development $^{30-32}$, as shown in this study. To systematically analyze the $A P 2$ family genes in pepper, the HMM profile with the AP2 domain (PF00847) was used as a query, and a total of $24 A P 2$ family genes were obtained in the genome database of the cultivated pepper Zunla-1 (Fig. 6). Duplication events are known to be the major driving force in the expansion of gene families ${ }^{33-35}$. To explore the major driving force for the expansion of the $A P 2$ family in pepper, the duplication types of each gene were detected by the MCScanX package, including singleton, dispersed, proximal, tandem and whole-genome duplication/triplication (WGD/T) or segmental duplication. Combined with the data of the retained triplicate genes from WGT in pepper ${ }^{36}, 50.0 \%$ (12) of the pepper AP2 family genes were assigned to $\mathrm{WGD} / \mathrm{T}$ or segmental duplication types (Fig. 6). This indicated that WGD/T or segmental duplication was the major force for the expansion of the AP2 family genes in pepper. Among them, the CaFFN (Capana02g003062) gene was located in a large collinear block containing 98 genes. The timing of this duplication event was estimated to be $\sim 47.31$ million years ago (Mya) based on the mean Ks value of segmental duplicated gene pairs within this collinear block. Thus, the segmental gene pair CaFFN and Capana02g000700 may have arisen from a WGT before pepper-tomato divergence (20 Mya) and after pepper-grape divergence (89 Mya) with a copy of the triplicated gene lost ${ }^{36}$.

To compare the expression patterns of the CaFFNrelated genes in pepper, Arabidopsis and tomato, the expression patterns of these genes in SAM at three different developmental stages were plotted in a heatmap (Fig. 6). Similar to the CaFFN gene, numerous CaFFNrelated genes showed a relatively high expression level at the vegetative meristem stage and a low expression level at the transition meristem stage, except for Capana04g002188, Capana09g001880, Capana10g001776, Capana10g001789, Capana11g000645, and TOE3.

\section{Discussion}

In this study, the locus controlling flowering time in the $F_{2}$ population derived from an intraspecific cross between the early flowering pepper $\mathrm{B}_{9431}$ and the late-flowering pepper $A_{145}$ was mapped to the end of chromosome 2 . This locus was reported to be a flowering time locus in several previous reports ${ }^{17,20,25}$. Borovsky et al. ${ }^{20}$ provided supporting genetic evidence for the putative function of CaAP2 in this chromosome region as a flowering repressor, but direct proof of the function of $\mathrm{CaAP} 2$ is still lacking. The CaAP2 gene (referred to in this study as the CaFFN gene) with a spontaneous natural point mutation $(\mathrm{SNP} 2 \mathrm{~T}>\mathrm{C})$ at the start codon in $\mathrm{B}_{9431}$ cosegregated with the FFN trait in the $F_{2}$ population of 297 individuals; this is additional genetic evidence for the function of CaFFN/ $C a A P 2$ as a flowering repressor. To provide more direct proof of the function of the CaFFN gene, transgenic $N$. benthamiana lines expressing the CaFFN gene were generated, and the flowering repressor function of this gene was verified by two $35 \mathrm{~S}:: \mathrm{CaFFNm} 3$ transgenic lines with a high level of CaFFN mRNA that flowered two and a half months later than control plants. The function of the CaFFN gene was also verified by VIGS of the CaFFN gene in the pepper $A_{145}$, which flowered earlier with a sixnode reduction in the number of FFNs.

Putative miR172 target sites in AP2-like genes are conserved in eudicots, monocots, and early land plants ${ }^{37-}$ 39 , indicating that the expression of AP2-like genes could be regulated by miR172. Mlotshwa et al. $^{38}$ generated transgenic $N$. benthamiana lines expressing Arabidopsis wild-type AP2 (35S::AP2) and the miR172-resistant AP2 mutant (35S::AP2m3). Nearly all $35 \mathrm{~S}:: A P 2$ plants were nonphenotypic with barely detectable levels of $A P 2$ mRNA; however, 35S::AP2m3 plants accumulated high levels of $A P 2$ mRNA and exhibited floral patterning defects such as the proliferation of numerous petals, stamens, and carpels. In our study, all 35S::CaFFN plants were nonphenotypic with a low level of CaFFN mRNA. However, only two $35 \mathrm{~S}: \mathrm{CaFFNm} 3$ plants that accumulated high levels of CaFFN mRNA exhibited an obvious late-flowering phenotype and floral patterning defects. It 


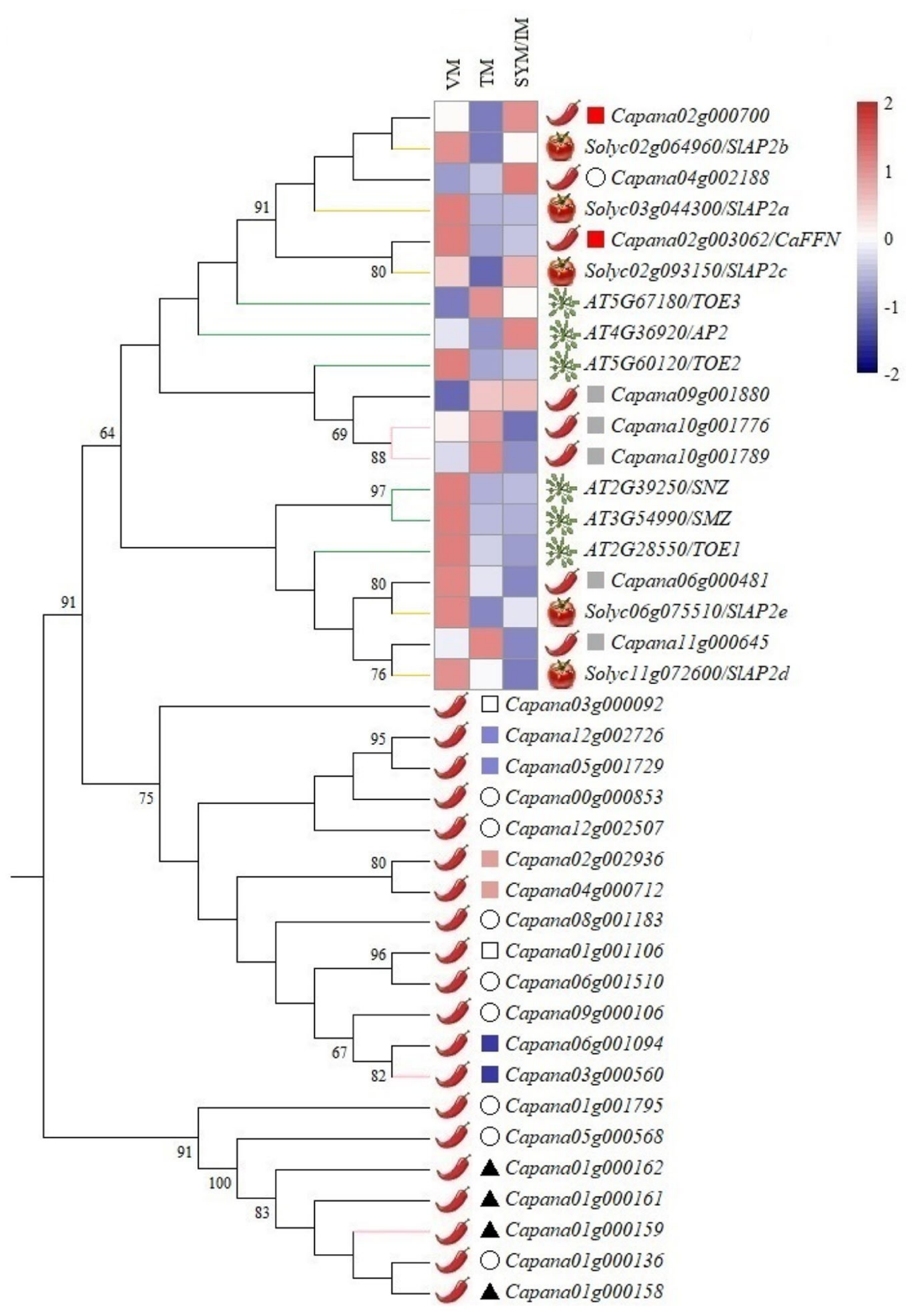

Fig. 6 Phylogenetic tree and expression heatmap of CaFFN/AP2 homologs. A phylogenetic tree was constructed by the maximum likelihood (ML) method; bootstrap values are based on 1000 replicates, and values lower than 60 are not shown; AP2 family genes and related genes in pepper are indicated with black branches and pink branches; CaFFN/AP2 homologs in tomato and Arabidopsis are indicated with yellow and green branches; dispersed, tandem, and whole-genome duplications/triplications (WGD/T) or segmental duplications are depicted by circles, triangles, and squares, respectively; duplicated gene pairs are indicated by squares in the same color, and retained genes with two copies of triplicated genes lost after triplication in pepper are indicated by hollow squares. An expression heatmap of 19 CaFFN/AP2 homologs was constructed, and the color scale at the top right represents the expression values scaled by the Pheatmap package (https://CRAN.R-project.org/package $=$ pheatmap) in R; the expression data of 19 CaFFN/AP2 homologs were obtained from this study and previous studies ${ }^{20,59,60}$. VM represents the vegetative meristem, TM represents the transition meristem, SYM represents the sympodial meristem and IM represents the inflorescence meristem

is inferred that a visible flowering-time alteration in $N$. benthamiana requires CaFFN mRNA to reach a certain threshold. Further analyses are needed to clarify the reason for the different expression levels of $35 \mathrm{~S}: \mathrm{CaFFNm3}$ plants. In contrast to $35 \mathrm{~S}:: A P 2 \mathrm{~m} 3$ plants with numerous petals, stamens, and carpels, these two 35S::CaFFNm3 plants exhibited short filaments, poorly developed petals, and a sterile phenotype. These results demonstrated that 
the CaFFN gene has a flowering repressor function as an AP2 gene in Arabidopsis and likely results in differentiation in the floral patterning of pepper.

In Arabidopsis, AP2 is involved in various developmental processes at the shoot apex ${ }^{40,41}$. The expression patterns of AP2 and CaFFN in SAM were consistent with their function as flowering repressors, which showed a high expression level at the vegetative meristem stage and a low expression level at the transition meristem stage. Similar expression patterns were found for nine additional homologs in Arabidopsis and tomato, indicating that these genes may have similar/overlapping functions. Previous research has demonstrated that six AP2 domaincontaining genes, including $A P 2$, showed functional redundancy of their role in flowering time in Arabidopsis; the early flowering phenotype of the $a p 2$ mutant was largely obscured by the effects of the other five members of the euAP2 lineage ${ }^{10}$. In contrast, all of the CaFFN mutants in C. annuum, including E- $62^{20}, \mathrm{~B}_{9431}, \mathrm{~B} 33, \mathrm{~B} 35$, and A100, exhibited an obvious early flowering phenotype, which indicated that the flowering time control function of this gene had little redundancy with other genes in C. annuum. This result was further confirmed by the expression patterns of CaFFN/AP2 homologs in the SAM of C. annuum, which differed from that of most of the detected CaFFN/AP2 homologs (Fig. 6), representing a functional differentiation of these homologs. On the other hand, no floral patterning defects were discovered in $\mathrm{E}-62, \mathrm{~B}_{9431}, \mathrm{~B} 33, \mathrm{~B} 35$, and $\mathrm{A} 100$, indicating that the function of floral patterning regulation of the CaFFN gene was redundant and could be compensated by other gene (s) in pepper.

Capana02g000700, as a paralog of CaFFN that has arisen from an ancient WGT ( 47.31 Mya), might have some functional redundancy with CaFFN since it presented a similar expression pattern as CaFFN in the SAM of pepper (Fig. 6). The possible function of Capana02g000700 in flower development and transcriptional repression of flowering time was also reported in a previous study ${ }^{26}$. However, Capana02g000700 may only have a weak effect on flowering repressor in C. annuum, since its expression level in the early flowering inbred line CA1 (C. annuum) was much lower than that in the late flowering inbred line 740 (C. chinense $)^{26}$. This is a reasonable explanation for why the early flowering phenotype of the CaFFN mutants in $C$. annuum was not obscured by Capana02g000700.

To investigate how the CaFFN gene participates in the complicated network to affect flowering time and floral organ morphogenesis, the transcription profiles of several key flowering time and organ development genes were assayed by qRT-PCR. The results suggested that CaFFN controls flowering time and floral development by regulating genes that are involved in the aging pathway (Fig. 4b): CaFFN functions as a transcriptional repressor to suppress the expression of the floral activator genes CaAP1, CaSEP3, and CaSOC1 as well as the key floral identity gene $\mathrm{CaAG}$; it also functions as a transcriptional activator to activate the expression of the floral repressor gene CaAGL15. In addition, CaFFN negatively regulated $m i R 172$ and positively regulated $m i R 156$, as indicated by their expression relationship. Moreover, yeast one-hybrid assays and dual-luciferase reporter assays revealed that CaFFN directly activates the floral repressor gene CaAGL15. These results provide the first insight into the mechanisms employed by CaFFN to regulate flowering time and floral patterning in pepper.

Genome-wide association analysis (GWAS) of 36 agronomic traits, including the FFN trait, in 287 pepper accessions was carried out based on SLAF-seq data, but no peak regions associated with the FFN trait were detected $^{22}$. It is unclear whether the CaFFN gene has a broad effect on flowering time in the pepper population. In our study, CaFFN gene association analysis was performed in 167 pepper inbred lines. A SNP 50 bp upstream of the start codon was significantly associated with the number of FFNs, explaining $14.5 \%$ of the phenotypic variation. This result indicated that the CaFFN gene has a broad effect on flowering time in the pepper population. This broad effect is not related to the SNP2T > C mutation at the start codon of $C a F F N$, which is a rare mutation in the pepper population, but is most likely due to the variation(s) in the $5^{\prime}$-UTR of the CaFFN gene. In addition to the result that the SNP in the $5^{\prime}$-UTR (SNP-50G > A) of CaFFN was significantly associated with the FFN trait, the results that SNP-50G > A significantly correlated with the expression level of the CaFFN gene indicated by qRTPCR also support the speculation that the $5^{\prime}$-UTR may influence the number of FFNs by affecting the expression of the CaFFN gene in the pepper population. Whether SNP-50G $>$ A is the causative mutation that influences the expression of CaFFN or a SNP tightly linked with the causative mutation needs further research.

The control of flowering time in pepper is an attractive objective for breeding aimed at creating locally adapted cultivars. In pepper, several genes, including $C a J, C a B L$, $C a S, F A$, and $C a A P 2^{14,15,19-21}$, were reported to be flowering regulators. Only $F A$ and $C a A P 2 / C a F F N$ were described as flowering repressor genes, and the effect of the $F A$ gene on flowering time was minor ${ }^{20,21}$. Despite a certain degree of reduction in shoot and fruit size and the extreme flowering time phenotype in the homozygous mutant of the CaFFN gene ${ }^{20}$, it is still an ideal breeding material for genetic improvement. Mutated CaFFN could significantly reduce the number of FFNs with minimal negative impact on yield in multiple genetic backgrounds when heterozygous, making it very useful for hybrid pepper breeding. This gene was used to select early 
maturing pepper varieties in our pepper breeding experiments.

\section{Materials and methods Plant materials}

The precocious pepper $B_{9431}$ was crossed with the lateflowering inbred line $\mathrm{A}_{145}$ (from the Asian Vegetable Research and Development Center). The $F_{1}$ hybrid was selfed to generate a segregating $F_{2}$ population. A total of 297 individuals of the $F_{2}$ population were used for mapping. The flowering time of pepper was measured by counting the number of leaves on the primary stem between the cotyledon and first flower. All plants were grown in a greenhouse in Nanchang $\left(28^{\circ} 33^{\prime} \mathrm{N}, 115^{\circ} 56^{\prime} \mathrm{E}\right.$, China).

\section{BSR-seq}

BSR-Seq combined bulked-segregant analysis and RNASeq and was performed to map loci for target traits. For the genetic analysis, two pools were constructed; each pool contained 30 individuals from the $F_{2}$ segregating population with extreme phenotypes. For the phenotyping of the FFN trait, total RNA of leaves at the blooming period was extracted using RNAiso Plus reagent (Takara, Dalian, China). RNA was quantified and assessed using a Qubit Fluorometer (Invitrogen, Carlsbad, USA), Nanodrop spectrophotometer (Thermo Fisher Scientific, Wilmington, USA) and Agilent 2100 bioanalyzer (Agilent Technologies, Palo Alto, USA). Paired-end sequencing was carried out on an Illumina HiSeq X-ten instrument. Clean RNA-seq data were mapped to the $C$. annuum cv. CM334 genome chromosomes (release 1.55, http:// peppergenome.snu.ac.kr/ $)^{42}$ using BWA software ${ }^{43}$. SNPs were screened using SAM tools ${ }^{44}$. The differences in allele frequencies of the 12 pepper chromosomes between the two pools were calculated and plotted.

\section{SNP marker development and genotyping}

To develop a SNP marker for the CaFFN gene, which was speculated to be the candidate gene controlling flowering time in pepper, the genomic sequences of the CaFFN gene in $\mathrm{B}_{9431}$ and $\mathrm{A}_{145}$ were amplified (with primers listed in Supplementary Table S1) and sequenced. The polymorphic SNP $(\mathrm{SNP} 2 \mathrm{~T}>\mathrm{C})$ at position 2 of the CaFFN gene between the $\mathrm{B}_{9431}$ and $\mathrm{A}_{145}$ genotypes was converted into the CAPS marker CSF2 for genotyping. PCR was carried out in $B_{9431}, A_{145}$, the $F_{1}$ population, and the $F_{2}$ population (297 individuals), and each reaction had a total volume of $10 \mu \mathrm{l}$ containing $50 \mathrm{ng}$ genomic DNA, $0.2 \mu \mathrm{M}$ primer FFN1-5UF4, $0.2 \mu \mathrm{M}$ primer FFN1-e1R2 (Supplementary Table S1) and $5 \mu \mathrm{l} 2 \times$ Taq Master Mix (Novoprotein Scientific Inc., Shanghai, China). PCR was performed with the following conditions: denaturing for $1.5 \mathrm{~min}$ at $94{ }^{\circ} \mathrm{C}$, followed by 38 cycles of at $94{ }^{\circ} \mathrm{C}$ for $20 \mathrm{~s}$, $55^{\circ} \mathrm{C}$ for $20 \mathrm{~s}$, and $72{ }^{\circ} \mathrm{C}$ for $1 \mathrm{~min}$, and a final elongation at $72{ }^{\circ} \mathrm{C}$ for $5 \mathrm{~min}$. The amplified PCR products were digested with the restriction enzyme TaiI (Thermo Fisher Scientific, Waltham, USA) directly in a final volume of $10 \mu \mathrm{l}$ consisting of $0.65 \mu \mathrm{l} 10 \times \mathrm{R}$ Buffer, $3.5 \mu \mathrm{l}$ PCR pro-

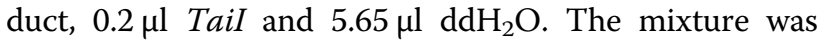
incubated at $65^{\circ} \mathrm{C}$ for $15 \mathrm{~h}$ and $80^{\circ} \mathrm{C}$ for $20 \mathrm{~min}$ and subjected to gel electrophoresis on a $2 \%$ agarose gel.

\section{Plant transformation of $N$. benthamiana}

CaFFN gene overexpression vectors were generated by cloning into the $\mathrm{XhoI}+\mathrm{XbaI}$ digestion sites of the vector pHellsgate 8. In this study, two different overexpression vectors were constructed: one included the wild-type CaFFN cDNA of $\mathrm{A}_{145}$ (35S::CaFFN), and the other included mutant CaFFN cDNA with six mismatches to miR172 that did not change the amino acid sequence (synonymous mutations) (35S::CaFFNm3). The recombinant plasmid was introduced into $N$. benthamiana by Agrobacterium-mediated transformation as described previously ${ }^{45}$. The plants were screened in medium supplemented with $75 \mathrm{mg} / \mathrm{L}$ kanamycin (the negative control could not regenerate) in a plant growth chamber at $24^{\circ} \mathrm{C}$ under $16 \mathrm{~h}$ light $/ 8 \mathrm{~h}$ dark cycles. The transgenic seedlings were identified by the primers FFN-RT-F and HR-gate8RV (Supplementary Table S1).

\section{VIGS in pepper $A_{145}$}

For CaFFN silencing analysis, the specific coding regions of the CaFFN gene were used for VIGS vector construction. The sequence specificity was confirmed by genome-wide homologous sequence searching by BLAST against sequences in the Zunla databases (http:// peppersequence.genomics.cn/page/species/blast.jsp).

Two fragments of $\sim 200 \mathrm{bp}$ in different regions of the CaFFN gene were inserted into the pTRV2 vector at the EcoRI site to construct two vectors, TRV2::CaFFN-1 and TRV2::CaFFN-2. In addition, a fragment of $\sim 100$ bp of the CaPDS gene was inserted into pTRV2 to construct a positive control vector, TRV2::CaPDS. The resulting vectors TRV2::rec and TRV1 were transformed into $A$. tumefaciens (strain GV3101). A. tumefaciens cells harboring TRV1 and TRV2::CaFFN-1/TRV2::CaFFN-2, TRV2 (as a negative control) or TRV2::CaPDS, as a positive control (resuspended in induction medium at a $1: 1$ ratio, $\mathrm{OD}_{600}=0.6$ ), were coinfiltrated into seedlings of pepper $\mathrm{A}_{145}$ at the four-leaf stage. The infiltrated seedlings were grown in a phytotron in the dark for $24 \mathrm{~h}$ and then at $20 \pm 1{ }^{\circ} \mathrm{C}$ under $16 \mathrm{~h}$ light $/ 8 \mathrm{~h}$ dark cycles with a relative humidity of $\sim 90 \pm 5 \%$.

\section{Quantitative reverse transcription PCR}

To determine the relative transcription levels of selected genes, total RNA was extracted from plant tissue using 
RNAiso Plus reagent (Takara, Dalian, China) according to the manufacturers' instructions. Single-stranded cDNA was synthesized using TransScript ${ }^{\circledR}$ II One-Step gDNA Removal and cDNA Synthesis SuperMix (TransGen Biotech., Beijing, China) for quantitative reverse transcription PCR (qRT-PCR) analysis of target genes or using TransScript $^{\circledR}$ miRNA First-Strand cDNA Synthesis SuperMix (TransGen Biotech., Beijing, China) for qRT-PCR analysis of target microRNAs. Quantitative real-time PCR was performed on a Bio-Rad CFX Connect Real-Time System (Bio-Rad Laboratories, Hercules, USA) using NovoStart ${ }^{\circledR}$ SYBR qPCR SuperMix Plus (Novoprotein Scientific Inc., Shanghai, China). Gene expression was calculated relative to CaGAPDH or CaU6 in pepper or relative to $N b b T U B$ in tobacco by the $2^{-\Delta \Delta C T}$ method $^{46}$. The amplification program was performed at $95^{\circ} \mathrm{C}$ for $2 \mathrm{~min}$, followed by $95^{\circ} \mathrm{C}$ for $10 \mathrm{~s}$ and $60^{\circ} \mathrm{C}$ for $30 \mathrm{~s}$ ( 45 cycles). The primers used for qRT-PCR are listed in Supplementary Table S1. In each case, three technical replications were performed for each of at least three independent biological replicates.

\section{Yeast one-hybrid $(\mathrm{Y} 1 \mathrm{H})$}

To investigate the interaction between the CaFFN transcription factor and CaAGL15, the full-length CaFFN open reading frame (ORF) sequence was transformed into the pGADT7 vector to provide the prey protein, and the cis-elements ( 2000 bp upstream of the start codon isolated from pepper $\mathrm{A}_{145}$ ) of the CaAGL15 gene were inserted into the pAbAi vector as bait sequences. The linearized bait plasmid pAbAi-CaAGL15 was transformed into the $\mathrm{Y} 1 \mathrm{H}$ Gold yeast strain, and the minimal inhibitory concentration of AbA was determined for the bait strain with the empty pGADT7 vector. Then, the prey plasmid pGADT7-CaFFN was transformed into the bait strain to detect the DNA-protein interaction by incubating them at $30^{\circ} \mathrm{C}$ for 3 days on $\mathrm{SD} /$-Leu medium supplemented with the minimal inhibitory concentration of AbA.

\section{Dual-luciferase reporter assay}

The cis-element of CaAGL15 ( $2000 \mathrm{bp}$ upstream of the start codon isolated from pepper $\mathrm{A}_{145}$ ) was inserted into pGREEN0800-LUC ${ }^{47}$ to generate the reporter plasmid pGreen0800-CaAGL15. The full-length ORF of CaFFN was inserted into the pGreenII 62-SK vector to generate the effector vector. The effector and reporter Agrobacterium cultures were mixed together and infiltrated into $N$. benthamiana leaves ${ }^{48}$. Empty pGreenII 62SK (EV) was cotransformed with the CaAGL15 reporter as a no-interaction control. Firefly luciferase and Renilla luciferase were quantified at 3 days after infiltration using Dual Luciferase Reporter Assay System reagents (Promega, Fitchburg, United States). Three biological replicates were conducted for each combination.

\section{Association analysis of CaFFN genes}

A forward primer FFN-solcs-5UF1 and a reverse primer FFN-solcs-e4R1 (Supplementary Table S1) were used to amplify a fragment of the CaFFN gene in 164 inbred lines from core pepper collections. Each amplified fragment was sequenced using the FFN-solcs-5UF1 primer, and the sequences obtained were aligned using MUSCLE ${ }^{49}$. SNPs and InDels were identified, and variants were filtered according to the MAF (MAF $\geq 5 \%$ ). The variants from 164 pepper inbred lines and FFN phenotypes were used in the association analysis, which was conducted using the cMLM in TASSEL v3.0 ${ }^{50}$. The population structure $(\mathrm{Q}$ matrix) and familial kinship (K matrix) derived from our previous studies ${ }^{51}$ were taken into account to avoid spurious associations. The associated variant(s) with the FFN trait were identified at the probability level of 0.01 .

\section{Analysis of $A P 2$ family genes}

The genome database of the cultivated pepper Zunla$1^{36}$ was used for the identification of AP2 family genes. The hidden Markov model (HMM) profile of the AP2 domain (Pfam Accession No. PF00847) was used as a query to BLAST against the Zunla-1 protein database. AP2 family members were identified by checking gene structures in Pfam ${ }^{52}$ to confirm the presence of two or more AP2 domains ${ }^{31,53}$. BLASTP alignment $(E<1 \mathrm{e}-5$, top five matches) was carried out across the wholegenome protein sequences, and the output results were loaded into MCScanX (MATCH_SIZE: 5$)^{54}$ to identify collinear blocks and to distinguish the singleton, dispersed, proximal, tandem, and WGD/segmental duplication types of these genes. The coding sequences of the AP2 family genes and four additional genes (Capana01g000159, Capana10g001789, Capana03g000560, and Capana10g001776) were used to create a codon alignment by DAMEB6 ${ }^{55}$. A phylogenetic tree of CaFFN/AP2 homologs was constructed using the maximum likelihood (ML) method in MEGA6 ${ }^{56}$, and branch reliability was assessed with 1000 bootstrap replications. The Ks (synonymous substitutions per site) values were calculated in PAML ${ }^{57}$. The formula $\mathrm{T}=\mathrm{Ks} / 2 \mathrm{R}$ was used to estimate the divergence time, where $\mathrm{R}$ is $1.5 \times 10^{-8}$ synonymous substitutions per site per year ${ }^{58}$. The expression data of 19 CaFFN/AP2 homologs were obtained from this study (primers are listed in Supplementary Table S1) and previous studies ${ }^{20,59,60}$. The expression data were scaled to construct the heatmap by the Pheatmap package (https://CRAN.R-project.org/ package $=$ pheatmap) in $R$.

\section{Acknowledgements}

This research was supported by the National Natural Science Foundation of China (31660574), China Postdoctoral Science Foundation (2020M671969), Agricultural Collaborative Innovation Project of Jiangxi Province of China (JXXTCXQN202001), China Agriculture Research System (CARS-24-G-08), and 
Key Research and Development Program of Jiangxi Province of China (20202BBF62002).

\section{Competing interests}

The authors declare no competing interests.

Supplementary information The online version contains supplementary material available at https://doi.org/10.1038/s41438-021-00643-7.

Received: 1 December 2020 Revised: 7 June 2021 Accepted: 13 June 2021 Published online: 01 November 2021

\section{References}

1. Henderson, I. R. \& Dean, C. Control of Arabidopsis flowering: the chill before the bloom. Development 131, 3829-3838 (2004)

2. Richter, R. et al. Floral regulators FLC and SOC1 directly regulate expression of the B3-type transcription factor TARGET OF FLC AND SVP 1 at the Arabidopsis shoot apex via antagonistic chromatin modifications. PLoS Genet. 15, e1008065 (2019).

3. MacAlister, C. A. et al. Synchronization of the flowering transition by the tomato TERMINATING FLOWER gene. Nat. Genet. 44, 1393-1398 (2012).

4. Fornara, F., de Montaigu, A. \& Coupland, G. SnapShot: control of flowering in Arabidopsis. Cell 141, 550 (2010).

5. Blazquez, M. A. \& Weigel, D. Integration of floral inductive signals in. Arabidopsis. Nat. 404, 889-892 (2000).

6. Moon, J. et al. The SOC1 MADS-box gene integrates vernalization and gibberellin signals for flowering in Arabidopsis. Plant J. 35, 613-623 (2003).

7. Liu, C. et al. Direct interaction of AGL24 and SOC1 integrates flowering signals in Arabidopsis. Development 135, 1481-1491 (2008).

8. Lee, J. \& Lee, I. Regulation and function of SOC1, a flowering pathway integrator. J. Exp. Bot. 61, 2247-2254 (2010).

9. Kardailsky, I. et al. Activation tagging of the floral inducer. Ft. Sci. 286, 1962-1965 (1999).

10. Yant, L. et al. Orchestration of the floral transition and floral development in Arabidopsis by the bifunctional transcription factor APETALA2. Plant Cell 22 2156-2170 (2010)

11. Abelenda, J. A., Navarro, C. \& Prat, S. Flowering and tuberization: a tale of two nightshades. Trends Plant Sci. 19, 115-122 (2014).

12. Samach, A. \& Lotan, H. The transition to flowering in tomato. Plant Biotechnol. 24, 71-82 (2007).

13. Weng, L., Bai, X. D., Zhao, F. F., Li, R. \& Xiao, H. Manipulation of flowering time and branching by overexpression of the tomato transcription factor SIZFP2. Plant Biotechnol. J. 14, 2310-2321 (2016).

14. Cohen, O., Borovsky, Y., David-Schwartz, R. \& Paran, I. CaJOINTLESS is a MADSbox gene involved in suppression of vegetative growth in all shoot meristems in pepper. J. Exp. Bot. 63, 4947-4957 (2012).

15. Cohen, O., Borovsky, Y., David-Schwartz, R. \& Paran, I. Capsicum annuum S (CaS) promotes reproductive transition and is required for flower formation in pepper (Capsicum annuum). N. Phytol. 202, 1014-1023 (2014).

16. Mimura, Y., Minamiyama, Y., Sano, H. \& Hirai, M. Mapping for axillary shooting, flowering date, primary axis length, and number of leaves in pepper (Capsicum annuum). J. Jpn. Soc. Hort. Sci. 79, 56-63 (2010).

17. Tan, S. et al. Construction of an interspecific genetic map based on InDel and SSR for mapping the QTLS affecting the initiation of flower primordia in pepper (Capsicum spp.). PLoS ONE 10, e0119389 (2015).

18. Zhang, X. F. et al. Candidate genes for first flower node identified in pepper using combined SLAF-seq and BSA. PLOS ONE 13, e0194071 (2018).

19. Jeifetz, D., David-Schwartz, R., Borovsky, Y. \& Paran, I. CaBLIND regulates axillary meristem initiation and transition to flowering in pepper. Planta 234 1227-1236 (2011)

20. Borovsky, Y., Sharma, V. K., Verbakel, H. \& Paran, I. CaAP2 transcription factor is a candidate gene for a flowering repressor and a candidate for controlling natural variation of flowering time in Capsicum annuum. Theor. Appl. Genet. 128, 1073-1082 (2015).

21. Elitzur, $T$. et al. Co-ordinated regulation of flowering time, plant architecture and growth by FASCICULATE: the pepper orthologue of SELF PRUNING. J. Exp. Bot 60, 869-880 (2009).
22. $\mathrm{Wu}, \mathrm{L}$. et al. Genome-wide correlation of 36 agronomic traits in the 287 pepper (Capsicum) accessions obtained from the SLAF-seq-based GWAS. Int. J. Mol. Sci. 20, 5675 (2019)

23. Barchi, L., Lefebvre, V., Sage-Palloix, A. M., Lanteri, S. \& Palloix, A. QTL analysis of plant development and fruit traits in pepper and performance of selective phenotyping. Theor. Appl. Genet. 118, 1157-1171 (2009).

24. Alimi, N. A. et al. Multi-trait and multi-environment QTL analyses of yield and a set of physiological traits in pepper. Theor. Appl. Genet. 126, 2597-2625 (2013).

25. Zhang, X. F. et al. High-density genetic map construction and QTL mapping of first flower node in pepper (Capsicum annuum L.). BMC Plant Biol. 19, 167 (2019).

26. Zhu, Z. et al. Construction of a high density genetic map of an interspecific cross of Capsicum chinense and Capsicum annuum and QTL analysis of floral traits. Sci. Rep. 9, 1054 (2019)

27. Anbessa, Y., Warkentin, T. Vandenberg, A. \& Ball, R. Inheritance of time to flowering in chickpea in a short-season temperate environment. J. Hered. 97, 55-61 (2006).

28. Boni, T. A., Prioli, A. J., Prioli, S. M. A. P., Lucio, L. C. \& de Mello, R. Inheritance of aluminum tolerance in maize. Crop Breed. Appl. Biot. 9, 147-153 (2009).

29. Ou, L. J. et al. Pan-genome of cultivated pepper (Capsicum) and its use in gene presence-absence variation analyses. N. Phytol. 220, 360-363 (2018).

30. Chung, M. Y. et al. A tomato (Solanum lycopersicum) APETALA2/ERF gene, SIAP2a, is a negative regulator of fruit ripening. Plant J. 64, 936-947 (2010).

31. Zhao, Y. et al. Genome-wide identification and analysis of the AP2 transcription factor gene family in wheat (Triticum aestivum L.). Front. Plant Sci. 10, 1286 (2019).

32. Lee, D. Y. \& An, G. Two AP2 family genes, supernumerary bract (SNB) and Osindeterminate spikelet 1 (OSIDS1), synergistically control inflorescence architecture and floral meristem establishment in rice. Plant J. 69, 445-461 (2012).

33. Maher, C., Stein, L. \& Ware, D. Evolution of Arabidopsis microRNA families through duplication events. Genome Res. 16, 510-519 (2006).

34. De Grassi, A., Lanave, C. \& Saccone, C. Genome duplication and gene-family evolution: the case of three OXPHOS gene families. Gene 421, 1-6 (2008)

35. Qiao, X. et al. Different modes of gene duplication show divergent evolutionary patterns and contribute differently to the expansion of gene families involved in important fruit traits in pear (Pyrus bretschneideri). Front. Plant Sci. 9, 161 (2018).

36. Qin, C. et al. Whole-genome sequencing of cultivated and wild peppers provides insights into Capsicum domestication and specialization. Proc. Nat Acad. Sci. U. S. A. 111, 5135-5140 (2014).

37. Aukerman, M. J. \& Sakai, H. Regulation of flowering time and floral organ identity by a MicroRNA and its APETALA2-like target genes. Plant Cell $\mathbf{1 5}$ 2730-2741 (2003)

38. Mlotshwa, S., Yang, Z., Kim, Y. \& Chen, X. Floral patterning defects induced by Arabidopsis APETALA2 and microRNA172 expression in Nicotiana benthamiana. Plant Mol. Biol. 61, 781-793 (2006).

39. Axtell, M. J. \& Bartel, D. P. Antiquity of microRNAs and their targets in land plants. Plant Cell 17, 1658-1673 (2005).

40. Wurschum, T., Gross-Hardt, R. \& Laux, T. APETALA2 regulates the stem cell niche in the Arabidopsis shoot meristem. Plant Cell 18, 295-307 (2006).

41. Ohto, M. A., Fischer, R. L., Goldberg, R. B., Nakamura, K. \& Harada, J. J. Control of seed mass by APETALA2. Proc. Natl Acad. Sci. U. S. A. 102 3123-3128 (2005).

42. Kim, S. et al. Genome sequence of the hot pepper provides insights into the evolution of pungency in Capsicum species. Nat. Genet. 46, 270-278 (2014).

43. Li, H. \& Durbin, R. Fast and accurate short read alignment with BurrowsWheeler transform. Bioinformatics 25, 1754-1760 (2009).

44. Li, H. et al. The sequence alignment/map format and SAMtools. Bioinformatics 25, 2078-2079 (2009)

45. Mlotshwa, S. et al. Transgenic plants expressing HC-Pro show enhanced virus sensitivity while silencing of the transgene results in resistance. Virus Genes $\mathbf{2 5}$ 45-57 (2002).

46. Livak, K. J. \& Schmittgen, T. D. Analysis of relative gene expression data using real-time quantitative $P C R$ and the $2^{-\triangle \triangle} \subset$ Method. Methods 25, 402-408 (2001).

47. Hellens, R. P. et al. Transient expression vectors for functional genomics, quantification of promoter activity and RNA silencing in plants. Plant Methods 1, 13 (2005).

48. Jian, W. et al. SIMYB75, an MYB-type transcription factor, promotes anthocyanin accumulation and enhances volatile aroma production in tomato fruits. Hortic. Res. 6, 22 (2019) 
49. Edgar, R. C. MUSCLE: multiple sequence alignment with high accuracy and high throughput. Nucleic Acids Res. 32, 1792-1797 (2004).

50. Bradbury, P. J. et al. TASSEL: software for association mapping of complex traits in diverse samples. Bioinformatics 23, 2633-2635 (2007).

51. Yuan, X. J. et al. Association analysis of resistance to Phytophthora capsici Leonian in pepper (Capsicum annuum L.) and exploration of elite alleles. J. Plant Genet. Resour. 20, 1026-1040 (2019).

52. El-Gebali, S. et al. The Pfam protein families database in 2019. Nucleic Acids Res. 47, 427-432 (2019).

53. Nakano, T., Suzuki, K., Fujimura, T. \& Shinshi, H. Genome-wide analysis of the ERF gene family in Arabidopsis and rice. Plant Physiol. 140, 411-432 (2006).

54. Wang, Y. P. et al. MCScanX: a toolkit for detection and evolutionary analysis of gene synteny and collinearity. Nucleic Acids Res. 40, e49 (2012).

55. Xia, X. DAMBE6: new tools for microbial genomics, phylogenetics, and molecular evolution. J. Hered. 108, 431-437 (2017).
56. Tamura, K., Stecher, G., Peterson, D., Filipski, A. \& Kumar, S. MEGA6: molecular evolutionary genetics analysis version 6.0. Mol. Biol. Evol. 30 2725-2729 (2013).

57. Yang, Z. PAML 4: phylogenetic analysis by maximum likelihood. Mol. Biol. Evol. 24, 1586-1591 (2007).

58. Koch, M. A., Haubold, B. \& Mitchell-Olds, T. Comparative evolutionary analysis of chalcone synthase and alcohol dehydrogenase loci in Arabidopsis, Arabis, and related genera (Brassicaceae). Mol. Biol. Evol. 17 1483-1498 (2000).

59. Park, S. J., Jiang, K., Schatz, M. C. \& Lippman, Z. B. Rate of meristem maturation determines inflorescence architecture in tomato. Proc. Natl Acad. Sci. U.S.A. 109, 639-644 (2012).

60. Winter, D. et al. An "Electronic Fluorescent Pictograph" browser for exploring and analyzing large-scale biological data sets. PLOS ONE 2, e718 (2007). 